\title{
Le Maroc entre la transition démocratique et une nouvelle renaissance socioculturelle
}

\author{
Bouazza Benachir*
}

\begin{abstract}
Résumé
Outre l'intégration du Maroc dans une vision mondialisée et respectueuse de la diversité culturelle qui caractérise ce pays, le paradigme de «Renaissance arabe» se doit d'être nuancé une fois rapporté aux socio-cultures marocaines. Ce double mouvement (mondialité et spécificité) corrèle avec la mise en perspective critique et théorique de l'entremêlement dialectique de trois horizons sociopolitique majeurs : la transition démocratique comme moyen et non une fin en soi ; la Région comme révélateur de l'efficace démocratique ; la diversité culturelle et humaine du Maroc. Le thème fédérateur de ce triptyque est la relation foncièrement politique existant entre la culture, la société et la démocratie et donc la charge sémantique et pratique de cette relation : les ethno ou socio-cultures sont indépassables quant à la question centrale du développement alternatif.
\end{abstract}

\begin{abstract}
Beside its role of integrating Morocco in a globalized vision that also takes into account the cultural diversity of the country, there is need for a nuance of the 'Arab Renaissance' paradigm in relation to the socio-cultural context of Morocco. This double movement (globality and specificity) correlates with the critical and theoretical perspective of the dialectic intermingling of three different major socio-political horizons: democratic transition as a means and not an end in itself; the region as an indicator of democratic efficiency; and Morocco's cultural and human diversity. The umbrella theme of these three elements is the unmitigated political relationship between culture, society and democracy

\footnotetext{
* Bouazza Benachir est Docteur d'État (Université Paris-I-Sorbonne) et ancien élève de l'ENA (École Nationale d'Administration, Paris). Chercheur à l'Institut des Études Africaines de l'Université Mohammed V-Souissi (Rabat, Maroc).
} 
and therefore the semantic and practical content of this relationship: ethnic or socio-cultural realities are indispensable to the central issue of alternative development.

\section{Introduction}

Trois perspectives retiendront notre attention à l'occasion de notre contribution aux travaux du thème de recherches sur «Repenser le développement africain : au-delà de l'impasse, les alternatives ».

1. Universaliser (mondialiser), dés-ethniciser, séculariser, etc., les approches de l'invention laïque d'une Renaissance marocaine diversaliste et autrement ouverte sur les exigences (politiques, juridiques, économiques, éthiques et culturelles) présentes et, à la fois, stratégiques du pays (hommes, cultures, démocratie territoriale ou interrégionale, recherche, éducation, développement alternatif...).

2.. Débloquer l'imagination créatrice tant individuelle que collective par la désaliénation tous azimuts et notamment par une réflexion critique sur le rapport de la politique à la question religieuse dont une des dimensions est le fascisme et $\boldsymbol{l} \boldsymbol{e}$ 《terrorisme ».

3. Initier, développer et promouvoir la diversité culturelle ${ }^{1}$ au Maroc, car ce pays est (au plan humain, linguistique et culturel) multiple. Autrement dit, il ne s'agit pas exclusivement de glorifier les dimensions euro-occidentale et moyen-orientale de ce pays, mais également ses dimensions amazighe (i.e. berbère) et négro-africaine et, donc, les régions (länder ?) qui composent ce pays.

Nous insisterons sur la dimension négro-africaine, car elle est peu connue des chercheurs marocains et africanistes : les Marocains noirs faisant partie intégrante des «fils aînés de la Terre » (Aimé Césaire à propos des Africains) et de la culture marocaine, il est indispensable de réhabiliter et de reconnaître leurs arts comme acteurs indépassables de la diversité humaine et culturelle du Maroc et, partant, comme porteurs de logiques métisses dont l'humanité aura de plus en plus vitalement besoin dans les temps présents et à venir. Le but visé par ce troisième axe de notre contribution, est de penser la diversité anthropo-culturelle en dehors de l'approche « réactive » (Nietzsche) et solipsiste du paradigme de l'ethnie dont les spécialistes savent qu'il peut être porteur d'une logique racialiste, voire génocidaire (Amselle 1990 ; Amselle et M'Bokolo 1999). Bref, de conflits et d'obstacles majeurs à la démocratisation des sociétés en voie de développement (en témoigne le cas catastrophique de la politique «négro-africaine » du Soudan à cet égard). 
Le champ interdisciplinaire ouvert et couvert par l'idée de «transition démocratique au Maroc » nous semble mériter d'être traité et approché à partir du triptyque dialogique et critique que nous proposerons, ici, à la méditation et au débat.

Ine transition démocratique et culturelle?

\section{Une transition politique?}

La transition démocratique ou l'«alternance consensuelle » (voir Herzenni 2002 : 25-32 ; Vairel 2001 : $301-15$; « La laïcité et la question de la transition démocratique au Maroc, Espaces consensuels » 2001, «L'agonie de la transition démocratique au Maroc» 2004) est une expression, certes inadéquate, mais sa généalogie est certainement très ancienne. La démocratie est toujours inachevée ; elle constitue un processus labile, adaptatif, souvent conflictuel et dialogique, et sans fin. Ab initio, il s'agit de la penser en en définissant la situation et la configuration dans un espace-temps donné pour en projeter et programmer les scansions qui ponctuent son déploiement. De fait, l'histoire sociale et politique du Maroc atteste que ce pays a vécu et connu, à l'instar d'autres nations, plusieurs types de transitions réussies ou avortées (Terrasse 1950 ; Laroui 1993 ; Allam 2005). L'essentiel est, donc, d'en saisir l'actualité en focalisant notre attention, serait-ce laconiquement, non sur les différentes périodes prises successivement ${ }^{2}$, mais, plus pragmatiquement, sur la période post-1999, car le référent philosophique et constitutionnaliste de cette dernière est les Droits de l'Homme et leurs corollaires : l'État de droit (sur cette notion voir Kahn 1989 : 49 et suiv.), la prise en charge des affaires de la Cité par le peuple.

Sans enter dans les détails, la cinquième période, l'actuelle, qui va de 1998-1999 à nos jours, est la période de restructuration et de refonte. Consensus et divergences en sont les marqueurs et les caractères manifestes. Le consensus est incarné par la nomination du gouvernement d'alternance, en 1998. La mission de celui-ci étant la mise en œuvre des principes universels de la démocratie dont le respect juridique des droits de la personne et des individus (corps, culture, désir, etc.). Les actions entreprises dans le sillage de cette mission continuent de faire l'objet d'évaluations et de lectures diverses, variées et contradictoires. Cette configuration n'est pas surprenante. Pour s'en rendre compte, il n'est que de pointer dans l'histoire passée et récente du Maroc, des faits qui 
font tantôt l'unité, tantôt la dissimilitude des forces politiques et socioculturelles qui co-existent dans le champ politique de ce pays. Il est des constats d'importance et à même de rationaliser le débat politico-social, culturel et économique au Maroc. C'est avant tout de la déconstruction-dépassement de ces données qu'il s'agit.

Premier constat : la Région. L'histoire du Maroc révèle une réalité plus que millénaire, à savoir que, - quoique dévoyée et sabordée par le caractère militariste de l'État marocain depuis au moins le $\mathrm{X}^{\mathrm{e}}$ siècle -, la démocratie régionale (Montagne 1989) est la première donnée du système politique de ce pays. Le deuxième constat coïncide avec l'attachement du pays à son unité nationale ${ }^{3}$.

Le troisième constat est à la base d'une conscience historique et collective tragique : le Maroc (hommes et socio-cultures) continue de payer, depuis plus de sept longs siècles infernaux, le prix exorbitant de tous les types possibles et imaginables (accommodés, troqués, organisés ou " réformistes $»^{4}$ ) de la sujétion politique (qui n'est pas qu'une « servitude volontaire »). Corollaire 1 : la formation schisée et schizante d'une culture de privilèges, de droit des hors-la-loi, et, donc, d'impunité outrecuidante - versus la plèbe et ses non-droits aux droits. Corollaire 2 : la confusion et l'indistinction de la ligne de partage séparant la sujétion et l'insoumission ${ }^{5}$

Quant au dernier constat, il porte sur les résultats auxquels aboutit un diagnostic clinique des données actuelles du Maroc politique. La configuration institutionnelle, culturelle et socio-économique, écologique, etc., du Maroc implique le rejet de toutes les formes de dogmatisme et de fanatisme. Corollaire 3 : une obligation d'innovation et d'immersion démocratique du peuple dans l'action politique, et ce, à la lumière de programmes inventifs, évaluables et mesurables. - La démocratie étant, à la fois, une utopie «actuelle » et un ressort, mieux : une ressource.

Compte tenu de la philosophie politique et de la globalisation des valeurs essentielles de la démocratie (Etchegoyen 1993, note 7), et à défaut de l'affirmation institutionnelle d'une culture de droits, parler de la démocratie au Maroc serait une billevesée tragique. L'affirmation et l'intériorisation d'une culture de droits est un problème institutionnel et, en même temps, socioculturel. Sans entrer dans les détails, la littérature relative à la démocratie et à la philosophie du droit consacre ceci, que le passage d'une culture de privilèges à une culture de droits est conditionné par la combinaison dynamique de plusieurs paramètres 
dont, entre autres, la redéfinition des rôles de l'État et des acteurs de la « gouvernementalité », l'indépendance de la justice et l'application canonique de la loi, sans aucune discrimination, l'accès citoyen et performatif au savoir, à l'éducation et à la culture, la prééminence de la dignité humaine dans l'approche de la double question cruciale du développement humain et de la participation plénière des Marocains à l'humanisation de la communauté du monde. La question culturelle, quant à elle, trouvera des issues bénéfiques si les acteurs (État, créateurs et écrivains, système éducatif et universitaire, partis politiques, société civile, etc.) prennent en compte, aux plans de la formation, de la sensibilisation et de la communication, une vérité socio-historique marocaine éminemment évidente, à savoir : la dimension diversaliste, plurielle et métisse du Maroc (hommes et cultures). Instituée par la plus haute instance de l'État marocain, l'INDH (Initiative Nationale du Développement Humain) vient à point nommé rappeler le caractère pertinent, et de cette donnée, et de l'un de ses pivots fondamentaux : soit la Région ${ }^{5}$ comme entité administrativo-économique, mais aussi comme support culturel et politique à même d'instaurer, d'un côté, l'aggiornamento des relations avec les «altérités » amazighe (« berbère), négro-marocaine et sahraouie, et, d'un autre côté, de révolutionner les « recompositions des modes d'accès à la sphère politique des différents acteurs et intérêts en présence » (Roussillon 2001 : 14), y compris les «élites locales» depuis longtemps considérées comme forces supplétives « autochtones » et désormais cataloguées comme «autochtones progressistes». Avec la mise en perspective pratique et institutionnelle de l'imbrication de cet aggiornamento (qui doit également englober les relations État-religion) et de ses recompositions, le Maroc ferait précisément de la transition démocratique une transition politique effective (Allam 2005).

\section{Le développement socioculturel au Maroc et l'obstacle des autochtones « orientalocentristes "}

Insérés dans des champs de savoirs différents et animés par des soucis épistémologiques et éthiques qui en font l'expression d'une histoire authentique du présent ou d'une critique archéologique du passé, au Maroc et depuis un siècle, tous les discours relatifs à la «transition démocratique » s'articulent au problème central auquel est confronté le champ politique marocain et, au premier plan, ses acteurs (État, gouvernement, parlement, partis, syndicats, société civile, mass-média, intellectuels, mouvements de défense des droits de l'homme, etc.). La 
préoccupation constante de ces acteurs consiste dans leur tendance commune à considérer prospectivement la (re)problématisation du paradigme d'État de droit : celui-ci, aussi bien que l'acte critique qui le pense (Laroui 1981), le « déconstruit », le donne à voir, etc., ressortissent de la décision consciente et vigilante de le situer et, en même temps, de l'instituer comme volonté de produire immédiatement une société, une socialité, une culture, une spiritualité, une grille de connaissance et un référentiel multipolaires : polyarchique (Ben Abdellah El Alaoui 2005 : 46-49). - En clair, le paradigme d'Etat de droit est condamné à être strictement fondé sur un socle constitutionnel dont le préambule et la finalité sont la démocratie (Rosanvallon 2002:16) ${ }^{6}$ et, donc, la renaissance ${ }^{7}$ socioculturelle et juridique d'une intellectualité et d'une praxis politiques qui dépassent, en le détruisant, l'assujettissement du citoyen (Laroui 1993 : 222-223, Leveau et al. 2004). Un tel chassé-croisé entre l'institution imaginaire du futur ou de la sauvegarde du possible, d'une part, et la prise en compte du réel (politique, droit, société, économie, égalité, citoyenneté...), d'autre part, révèle la présence active et inquiétante, tout à la fois, d'une ambivalence et d'une différence historico-existentielles inhérentes aux discours de Renaissance (discours du Même ou discours de l'Autre reproduisant - est-ce l'éternel retour ? - le Passé dans son impossibilité même ; discours de l'Autre, celui de la rupture critique, « Lumièriste », ou Aufkärer, avec l'archée et avec une recherche de soi narcissique mais immobilisante...) (Laroui 1987 : 127-151).

Dans ce sens, la «Renaissance » politique, comme objet théorique et comme préoccupation anthropo-historique, fonctionne comme analyseur et révélateur de la part d'insu contenu dans la Renaissance arabe (ou d'expression arabe) et ses discours. En effet, elle révèle comment la pensée et la conscience arabes modernes et contemporaines s'intensifient et progressent de manière significative dans l'ambition de «renattre » à mesure ou à chaque fois que se précise et s'approfondit la prise de conscience par les Arabes, par leurs cultures et leurs « horizons d'attente » (Jauss), de leur « déclin». Cette situation « logique» fait fond sur une différence : une différence entre un « déclin » palpable (palpable, le « déclin » en question l'est, puisqu'il fait partie du vécu arabe, de la quiddité arabe) et une « Renaissance arabe » glorifiée comme donnée réelle ou comme réel proposé aux Arabes et à leurs discours par deux références ou deux « méthodologies » ou encore deux « modèles » : d'un côté, le modèle arabo-islamique et ses diverses variantes (dont la 
version «islamiste ») ; de l'autre côté, le modèle européen inventeur et instaurateur de la modernité et de la « globalisation-mondialisation ».

Conséquence de cette idée de différence ou de cette situation ambivalente : penser la «Renaissance arabe » ne signifie pas la penser en tant qu'alternative à ce qui est ; penser cette Renaissance doit pouvoir objectivement exiger ce que voici : poser les conditions de possibilité d'une alternative produite à partir de ce qui existe effectivement. La « Renaissance » étant une praxis, et non plus une catharsis, seraitelle d'emprunt...

Assurément, l'ambivalence comportementale, discursive et cognitive de l'homo arabicus face à la Renaissance comme projet (et, à la fois, comme dépassement de cette ambivalence même) est le corollaire de ceci, que cet homme théorise la «Renaissance » en la décontextualisant, en la déréalisant (Tuéni 1991). Autrement dit, l'homo arabicus pense la Renaissance dans le cadre d'outils théoriques d'autant plus obsolètes (en dépit de leur apparence opérationnelle) qu'ils se traditionnalisent et que cet homme n'en est guère conscient quant à leur mode d'emploi dans l'accès, intelligent et positivement productif, au réel :

- l'homo arabicus pense la « Renaissance arabe » dans et à partir du modèle arabo-islamique qui, lui, s'enracine sans cesse dans le passé, dans l'Origine. Et en s'éloignant de plus en plus considérablement du passé, ce modèle ne permet pas que s'exerce à son propos une pensée objective ; pire : les faits objectifs sur lesquels s'établit ce modèle faisant défaut, toute volonté de le penser devient sans objet, voire l'objet d'une inquisition feutrée ou franchement catastrophique (Bekri 1994: $206)^{8}$.

- l'homo arabicus pense la «Renaissance arabe » dans et à partir du modèle européen. Mais à l'inverse du premier modèle (re)traditionaliste, le modèle européen s'enracine dans le temps présent; il se confond avec le futur; il produit le futur. Et toute volonté « nationalitaire » arabe d'être en synergie avec ce processus producteur d'avenir s'amenuise sous l'effet du gigantisme des progrès politiques, économiques, sociaux, scientifiques, télé-technologiques, culturels, etc., générés par ce modèle «dromologique » (du terme grec dromos qui signifie vitesse)

En fait, l'alternative consiste moins dans l'emprunt de l'un ou l'autre modèle, que dans l'analyse de la réalité du « déclin » (versus « renaissance »). C'est le fait de faire l'impasse sur la nécessité incontournable de cette analyse - analyse qui fait se conjoindre la volonté de Renaissance avec la conscience de l'urgence de la production-maintien de cette 
même volonté-, qui éclaire le pourquoi et le comment de l'« exigence » d'une «Renaissance arabe» autre. Mais cette « exigence» pourrait, d'une part, occulter le réel socio-historique et politique, et, d'autre part, déterminer idéologiquement le rapport du réel arabe en question avec l'un des deux modèles susmentionnés. En d'autres termes, le discours ou l'exigence de Renaissance, en optant pour ou en « intériorisant » l'un des modèles que voilà, est susceptible de l'utiliser comme théorie de couverture/légitimation ou comme écran qui occulte, précisément, le vécu multidimensionnel et la réalité économique et socio-politique des peuples arabes ou d'expression arabe comme le sont, on le sait, les peuples d'Afrique du Nord.

Les approches et les analyses contenues dans les «langages arabes $d u$ présent » (Berque 1974) convergent, chacune selon ses démarches propres, vers un point essentiel : à savoir que la représentation arabe de la Renaissance découle de la relation structurelle qui unit quatre composantes : le modèle européen, le modèle arabo-islamique, la question radicale du « déclin » et l'exigence philosophico-politique d'en sortir. En outre, la relation qui existe entre ses quatre facteurs se complexifie quand les sous-composantes (culturelle, « ethnique », sociale, scientifique, éthique, esthétique, économique, sociale, politique, juridique, stratégique, etc.) de chacun d'eux se mettent en rapport avec celles des trois autres ou inversement. De là, plus qu'à un « conflit des interprétations » (Paul Ricœur) de la nature du rapport en questions ; - au Maroc, par exemple, on est en présence d'une mise à nu salutaire des structures anthropologiques et logiques de la conscience socio-culturelle et politique contemporaine dans son désir besogneux de réaliser la Renaissance, hic et nunc.

De fait, la pensée arabe moderne et contemporaine s'articule, selon les promesses des thèses multiples qui la traversent, à la Renaissance du point de vue d'une double perspective : celle du recommencement et celle de la problématisation. Plus précisément, la Renaissance, pour la pensée arabe et ses différentes composantes, demeure un projet. Elle renvoie à une pensée du commencement et des limites (Nassar 1997: 57-80). Ces thèses rappellent, en substance, comment la problématisation de l'idée de Renaissance détermine, et ne cesse de le faire, le renouveau même de la pensée arabe et son arborescence en interprétations (souvent différentes, contradictoires, conflictuelles...) du passé, du présent et de l'avenir du Monde arabe et de ses cultures. 
Étant donné la configuration et la constellation politiques, idéologiques et culturelles qui déterminent de part en part le Monde arabe, il est canoniquement évident que si une Renaissance arabe nouvelle il y a, elle pourra un jour se faire avec les prophètes de Dieu (voir le cas de l'Algérie, de la Palestine, du Liban $)^{9}$, certes, mais aussi avec les prophètes des hommes et des nations. Cette dyarchie est d'autant plus compréhensible qu'elle fait fond sur la composition multiconfessionnelle et multiculturelle-multilinguistique des univers arabe ou d'expression arabe. C'est ce qu'occultent les propos intéressés et irrationnels de certains chercheurs maghrébins soucieux d'apparaître plus Arabes que les Arabes... ${ }^{10}$

\section{La région entre invention, création et résistances ${ }^{11}$}

Limportance théorique et pratique du paradigme institutionnel de la Région (Réforme Constitutionnelle de septembre 1996 et l'Initiative Nationale pour le Développement Humain - INDH - été 2005) tel qu'il est à l'œuvre au sein des discours officiels marocains, est d'autant plus claire qu'elle souligne le lien qui fait nationaliser ou trans-nationaliser ou encore métisser le rhizome culturel qu'est la diversité humaine à l'œuvre au Maroc.

Ce paradigme permet d'aborder la question du Maroc comme horizon culturel métissé. Le métissage étant une transformation, une métamorphose ou un basculement de l'identité dans la diversalité, la région est un des ressorts et un des supports essentiels de la pratique démocratique qui se base, elle, sur la transversalité-horizontalité et non plus exclusivement sur le centralisme-verticalité. Il n'est pas question d'opposer, ici, la diversité de la société à l'unitéisme de l'Etat. Le centralisme dit «démocratique » ayant fait l'expérience historique avortée qui fut la sienne, au siècle dernier, les acteurs de l'analyse et de la mise en ouvre du concept de la région se doivent d'être constamment attentifs à l'idée de délégation ou de décentration-déconcentration.

\section{La région : une ressource et un horizon diversaliste}

La décentration est au principe de la philosophie politique qui formule la définition de la région par les discours royaux précités. De même que la notion de transfert de souveraineté obéit rigoureusement à la représentation marocaine de l'État, de même la mise en perspective prospective de celui-ci ne déroge pas à cette règle. En d'autres termes, pas plus 
qu'il ne s'agit de « réinventer » notre État, il n'est question de «créer » la région. Comme l'histoire, les géographies (humaine et physique) fondent, en amont (État) et en aval (élus communaux, municipaux, provinciaux et régionaux, société civile), un Maroc où, à côté de la diversité bénéfique des régions, la diversalité des cultures est d'emblée une donnée structurelle et, à la fois, le produit d'une métamorphose socio-culturelle ou d'une acculturation historiquement et présentement attestées. Celles-ci sont intériorisées par la société et, en même temps, par l'État. L'histoire des mentalités l'atteste, à l'instar de l'anthropologie et de la sociologie politique, du reste.

Le paradigme régional est moins « régionaliste » ou administratif que juridique. Pratiquement et en principe, il devrait avoir comme support essentiel la souveraineté organique aux actions d'équité qu'elle (la souveraineté) met en œuvre au plan de tous les domaines nationaux et internationaux dans lesquels elle s'inscrit et s'incarne. Ces domaines, on comprendra qu'ils fassent l'objet d'une délibération institutionnelle et sociétale transversale mais orchestrée et modérée par l'État central. Ils n'auraient à être donc le fait ni des voix des «marges » (pourtant très actives) de la société, ni de ses «centres » autoproclamés comme tels et bénis par leurs connivences avec les pouvoirs des médias (et des appareils éducatifs) qui s'arrogent le droit (ou le non-droit) de dispatcher le peuple en deux catégories: l'« autochtone » et la « civilisée ». Cette ventilation délirante étant une des conséquences d'une philosophie du langage significativement naïve, parce que porteuse d'une illusion intéressée par la perpétuation des privilèges égocentriques qu'elle légitime : ce en quoi une telle « philosophie» est une idéologie... exogène, étrange, inquiétante, dangereuse pour le pluralisme politique et « ethno »-culturel du Maroc.

La construction de la région a une histoire. Celle-ci est pan-humaine. Seulement, avant que la région ne devienne un fait historique, elle aura d'abord été le produit d'un débat contextuel, c'est-à-dire situé dans un espace-temps institutionnel déterminé et précis. C'est pourquoi il y a autant de conceptions de la région qu'il y a de souverainetés nationales (USA, Royaume-Uni, Espagne, Allemagne, Suisse, Brésil, Mexique, Nigeria, etc.).

La théorie de la région dépasse donc le seul cadre économique, par exemple. Elle symbolise la mutation de la totalité de la société qui la produit et l'applique. Or, ici et maintenant, cette mutation aura à considérer, dialectiquement et pragmatiquement, certains aspects des com- 
plexités concrètes de nos sociétés contemporaines, c'est-à-dire : entre autres, la revendication et la recherche convulsive - par les individus « genrés » (différence des sexes), les groupes, les institutions, les organisations, les associations (dont les ONG), l'éducation, les cultures (langues, arts, mass-médias...), les « régions », les jeunes, etc. -, d'une multiplicité fondamentale. En d'autres termes, chacun de ces acteurs revendiquent et recherchent, chacun à sa manière et selon sa définition de la situation (région, société, État...), sa vérité, mais une vérité qui ne soit plus confisquée par les institutions-tampons qui sont chargées (par qui ?) d'être des médiateurs entre le symbole légitime de la souveraineté nationale et la multitude des acteurs que voilà.

\section{Région, être-en-commun et logiques de créativité}

Même fantomatique, tout comme tout homme (quels qu'en soient le sexe, les visions, les ambitions et les utopies) n'est pas sans moi, je ne puis exister sans lui. C'est cette dialectique qui est en jeu dans les coexistences métisses que la théorie institutionnelle de la Région postule comme horizon que les pratiques sociétiques sont capables d'atteindre. La Région représente en effet un enjeu stratégique: celui de la construction d'un être-ensemble basé sur la partage et la solidarité et, en même temps, sur la différence comme logique de créativité. Ce qui implique la construction d'une méthodologie et des mots nouveaux qui rompent avec la « langue de bois » qui aura longtemps oblitéré l'accès à la connaissance du Divers tel qu'il anime et travaille la société marocaine concrète.

Il s'agit d'aller au-delà des ombres des composantes molaires de la société marocaine. Ce qui spécifie la dimension diversaliste du Maroc, ce n'est pas le fait du métissage biologique et culturel (puisqu'il y en a partout chez les Marocains), mais la place que les «logiques métisses » occupent dans ce pays. Autrement dit, cette dimension est un témoignage vivant de l'Autre qui est organique aux tréfonds psychoculturels du Maroc métis. Cependant, encore faut-il que ce témoignage fasse événement théorique. C'est à conditionner le paradigme régional par l'avènement de cet événement que la Région, de l'un des critères de notre société plurielle qu'il est, pourrait faire partie intégrante des éléments de la grille d'évaluation de la mise en œuvre de nos valeurs ancestrales d'équité et de justice. Nous disons de nos valeurs ancestrales d'équité et de justice, car et notre société et son Etat son approchables (au sens de connaître) à partir de ces valeurs même. C'est la négation de 
celles-ci et, donc, la tentation de néantiser le socle archéologique, ancien, de notre société et de notre État, qui exigent une nouvelle lecture historiographique, socio-politique et juridique, de notre legs historique qui, lui, s'inscrit dans le temps présent et les temps immédiats et prospectifs, et corrèle, par conséquent, avec la nécessité de l'avènement d'une approche plus sereine des différents problèmes qui sont liés à l'idée de Région. Une évaluation plus réfléchie, plus anthropologique, voire psychanalytique, est envisageable qui étudie et analyse les effets « acculturels » des « résistances » à/contre l'idée démocratique-révolutionnaire de Région. Sans entrer dans les détails ni cultiver l'idée d'encerclement, ces résistances obéissent à des motivations diverses. De toute manière, elles sont extérieures et intérieures. En bref, elles impliquent leur dépassement et, partant, une révolution culturelle à l'espagnole ou la tchécoslovaque.

\section{La région alternative au centralisme}

Nul oubli n'est concevable, possible, dans ce vaste mais déterminant défi, à savoir celui de la postulation et de la construction de la Région (Sedrati 1995: 111-117). À l'instar du quasi-dévoiement de la règle démocratique par certains traitements courants et « habitudes » jupitériennes, la question (de cette nature) de la Région demeure omniprésente. Ce qui signifie que cette question (pas seulement elle) n'est toujours pas réglée ou, - puisque son règlement est enclenché par la dynamique socio-politique et culturelle nationale en cours -, qu'elle achopperait sur les « résistances » d'idéologies qui ne disent pas leurs noms et qui entravent la marche du Maroc vers la construction d'un rhizome plénièrement marocain mais néanmoins ouvert sur la communauté démocratique du Monde: soit un État qui n'est pas contre la société, ni contre les diverses composantes de celle-ci. En clair, l'existence de « résistances » à la Régionalisation renvoie au fait, majeur et stratégique, que, d'une part, la dynamique nationale actuelle en matière de « länderisation » est justifiée, et que, d'autre part, les traitements douteux de cette question sont devenus caducs. La question du Sahara occidental et la revendication amazighe sont des révélateurs majeurs de cette caducité incohérente.

$\mathrm{Au}$ Maroc, toutes les régions ont contribué à la constitution des logiques transculturelles nationales, et ce, sans que personne ne s'en rende compte. Cette donne s'explique par la fusion culturelle, cultuelle et spirituelle des Marocain(e)s; cette fusion favorisant (et continuant de 
favoriser) l'avènement d'une culture diversaliste et unitéiste, à la fois. Il est d'aucuns qui objecteront que la culture ne nourrit pas son homme, et que même en le nourrissant, elle est nécessaire à maintenir et à défendre, mais pas suffisante. Qu'est-ce qui est et nécessaire et suffisant, donc? L'équité et la justice?

\section{Équité et diversité humaine au service du développement humain et de la région}

Mais n'est-ce pas que la présente réflexion a d'entrée de jeu posée cette question? Récapitulons. La région comme paradigme moléculaire et polyarchique est de nature à mettre en ouvre la sauvegarde et le développement de la diversité humaine au Maroc.

Il est important de souligner ceci : la culture cosmopolite est une des fondations prioritaires du développement humain au sein des stratégies à venir de transferts d'hégémonie de créativité dans le cadre de la «Province » télé-techno-scientifique actuellement de plus en plus hégémonique à l'échelle mondiale.

Manifestement très actuel au Maroc (au niveau de l'État, des missions du système éducatif, et de la société civile), ce débat gagnerait en profondeur et en clarté, s'il s'articulait sur l'avènement, - constitutionnel et d'ores et déjà décennal -, dans ce pays, de la Région et, en même temps, sur la demande de reconnaissance des cultures « populaires » et « régionales » par les pouvoirs locaux et régionaux. L'existence du Sénat (la deuxième chambre du parlement) serait in-opérationnelle et dispendieuse si elle n'accompagnait pas de manière rigoureuse et efficiente la demande de régionalisation et les multiples défis que sa réalisation se doit de relever. Bien entendu, le gouvernement central n'est pas sauf de ses responsabilités et missions, autrement dit : pour que la « länderisation» ne soit pas un vain mot qui s'ajoute au slogan creux, il faut que l'Etat et ses organes concernés par cette problématique et à côté de la société civile, s'impliquent et s'investissent davantage et de manière stratégique et clairvoyante.

\section{Inventer la Région n’est pas la recréer}

C'est l'invention de la Région par les cultures et les individus concrets qui lui sont organiques, qui créera les Régions.

La présente contribution à un débat majeur devrait être relayée ou critiquée par d'autres approches car le pari est de déboucher sur la for- 
mulation de politiques sociales et culturelles de proximité recadrées par une lecture, in fine, des attendus d'une théorie rigoureuse, démocratique et sans exclusivisme de la Région. Il ne s'agit pas de débattre de ce que la Région peut nous donner, mais de ce que nous pouvons faire pour elle. Certes, elle est aussi un concept lié à la théorie de l'aménagement du territoire. Mais encore faut-il faire de nécessité vertu, autrement dit : aménager des espaces destinés aux sciences et aux techniques et aux savoir-faire capables de répondre aux demandes de nos régions.

Exemples : une ophtalmologie spécifique au désert ou au pré-désert; des sciences agronomiques adaptées à ces mêmes zones ainsi qu'aux zones arides ou semi-arides ou montagneuses ou encore aux littoraux. Trois autres exemples (de taille) : celui de l'eau, des transports (Qu'attend-on pour creuser un tunnel sous le Haut-Atlas Central ou réaliser l'extension des réseaux ferroviaires et auto routiers ?), celui de l'exploitation des richesses halieutiques, celui de l'éco-tourisme. Ce sont là des chantiers d'importance qui doivent être mis en œuvre car ils font partie intégrante de la démocratie territoriale et des politiques de désenclavement égalitaristes et d'intégration régionale et interrégionale. Va-t-on encore objecter que ces domaines et celui de l'« aménagement linguistique » du territoire, sont d'ordre « culturel »...? En bref, l'événement réaliste et efficace d'un aménagement du territoire en termes politiques, culturels et télé-techno-scientifiques, passe par l'instauration de régions autonomes et, en même temps, par celle d'un État décentrédécentralisé.

La démocratie n'est pas uniquement une fin, mais elle est aussi un moyen. Des pans entiers de l'humanité vivent à l'âge post-démocratique. La Région n'est l'apanage de personne, car il n'est pas encore prouvé qu'elle ne représente qu'une simple panacée.

Comme dirait le barbu de Trèves (il s'agit de Karl Marx), il s'agit moins de penser la région que de la créer. En reconnaissant officiellement l'état de précarité avancée accablant ses régions et ses « ethno-cultures » les plus enclavées et les plus démunies, le Maroc officiel ne peut que faire de nécessité vertu, c'est-à-dire : créer les conditions de possibilité d'une productivité conceptuelle et pratique capable de mettre à nu un des aspects des marges de son humanité socioculturelle et économique. Le vécu des communautés Noires du Maroc que nous présentons ciaprès n'est qu'un exemple parmi d'autres illustrant la précarité 
structurelle de plusieurs communautés marocaines rurales, urbaines, suburbaines, etc.

In cas de développement culturel alternatii :

la communauté noire d'Essaouira-Mogador (Maroc)

\section{Cité cosmopolite et Port de Tombouctou ou l'influence culturelle subsaharienne à Essaouira}

C'est de l'influence et du métissage culturel de ces Noirs sur, ou avec, les « autochtones blancs » que nous traitons dans ce qui suit, en termes d'ethnographie urbaine et d'ethnométhodologie (Schutz 1932, tr. fr. 1987 ; Garfinkel 1967 ; Quéré 1984 ; Coulon 1987).

Essaouira est la seule ville du Maroc où les Afro-Marocains ont construit, à la fin du XIX siècle, un sanctuaire, une zaouïa, celle de Sidi Bilâl. Ce sanctuaire se situe à côté de la Sqala (batterie de canons), le long de l'Océan atlantique. Ce sanctuaire est doté d'une école coranique; au fond du patio, on trouve quatre pièces de cérémonies rituelles.

À Essaouira, l'influence de la civilisation du Bilad es-Soudan (i.e. « Le Pays des Noirs ») est un tropicalisme urbain et esthétique transversal qui touche l'ensemble des composantes de la totalité des cultures qui y sont en interaction. Ce tropicalisme nourrit l'imaginaire des jeunes générations, et devient le creuset d'une culture capable de passer du localisme à l'universalité. Toutefois, si ce tropicalisme n'est supporté en surface que par une existence fantomatique, l'espace d'expression identitaire africain-marocain possède une consistance d'autant plus symboliquement forte que celle-ci est immanente à l'épaisseur de la mémoire collective marocaine et à ses strates souterraines oubliées, ou secrètement célébrées, comme l'attestent de nombreux travaux ethnologiques. Et cet espace en révèle un autre, occulté, qui devient une autre Afrique fantôme, pour parler avec Michel Leiris. Cette Afrique fantôme, c'est l'homme austral et en particulier les Gnawa-Ganga ; elle constitue la face cachée et, sans doute, l'envers de l'identité inter-ethnique originaire syncrétique d'Essaouira et, sans doute, du Maroc dans sa totalité.

Plusieurs thèmes peuvent ponctuer la progression de notre présente réflexion. L'un de ces thèmes consisterait, précisément, dans la contextualisation de cette réflexion, et dans sa restitution au contexte cultuel et culturel d'Essaouira. De telle manière seulement, notre approche pourrait donner à voir la part invisible ou le négatif de cette 
ville. Cette part est Autre et, en même temps, Même : voir la vicariance culturelle à Essaouira et, à la fois, analyser les mécanismes à travers lesquels l'ordre culturel noir occupe la place laissée vacante par les cultures «majoritaires» « autochtones». Il y a là comme un « retour du refoulé » interculturel et métissé : en ressortissant d'un espace urbain (Essaouira) auquel il participe, et que, en y participant, il révèle et en rend visible l'impensé (son origine négro-africaine), l'ordre culturel négromarocain local se maintient et s'affirme.

En effet, telle qu'elle s'est constituée à Essaouira, la culture urbaine est à lire à partir de la prévalence culturelle de l'élément Noir sur la pop'culture locale, puisqu'il n'y avait que lui, à côté des trois ordres constitués de la ville, au moment de sa construction et tout le long de la première moitié du XIX ${ }^{\mathrm{e}}$ siècle: à savoir les représentants de l'État central marocain, le personnel consulaire européen et les commerçants judéo-marocains.

Or, la présence et la fonction des expressions culturelles à Essaouira participent du paradigme africain de cette ville. Par paradigme africain, nous entendons désigner la production, consciente ou inconsciente, à travers les expressions picturales, musicales, rituelles, etc., de la quiddité (l'essence de la chose, selon les thomistes) ou de la spécificité d'Essaouira et, en même temps, de ses ethno-cultures transversales à son espace urbain.

Un des mouvements génésiques de l'importance de plus en plus affirmée de l'ordre culturel africain-marocain, c'est la rencontre de l'identité culturelle négro-marocaine avec les mouvements culturels planétaires désignés par les expressions de world music et de world culture (jazz, rythm and Blues, Living Theater, hip hop, ragamuffin, etc.), qui sont, comme on le sait, d'origine africaine-américaine. Loin de constituer uniquement un processus de « déterritorialisation » (Deleuze et Guattari 1980), cette rencontre culturelle pan-diasporique africaine avec « la mondialisation globalisée » des musiques du monde, a également déclenché le processus d'« altermondialisation » des expressions artistiques des Noirs d'Essaouira.

La fonction «symbolique » de ces Noirs se libère ainsi de son localisme et de sa dimension strictement rituelle pour acquérir progressivement et sûrement un statut esthétique qui s'agence avec le dehors, avec l'harmonie universelle en noir et blanc, pour parler avec Fourier. De même que leur musique, leur peinture, ici à Essaouira, produit le basculement de cette culture «mineure» dans l'universel ou, si l'on veut, 
dans les Ailleurs, dans les identités qui, pour cesser de fonctionner comme recherche mimétique de soi, s'agence comme singularité, ou ritournelle, avec la nécessité d'exister, certes, mais d'exister en tant qu'œuvre d'art...

La saisie de la signification du tournant esthétique en question est nécessaire. Car, à être analysé du point de vue de l'ethnographie urbaine, ce tournant constitue, aussi, un tournant socio-urbain. Non seulement ce tournant dé-ritualise les expressions culturelles noires d'Essaouira en les donnant à voir et à se produire comme spectacle, mais, plus encore, il instaure un Cogito de l'artiste d'obédience négro-africaine. Ce Cogito permettant à cet artiste d'affirmer son existence sociale plénière. C'est l'effet-Aufhebung (non obligatoirement hégélien !) : certes, le souvenir de l'esclavage persiste, mais il est transmué en corybantes dionysiaques qui permettent à la honte d'être soi (Noir, donc « esclave») de devenir une ressource, voire une puissance, tout à la fois ontologique, existentielle et sociale. L'énoncé du Cogito que voilà pourrait être formulé de la manière suivante: Ce que je joue, comme musicien africain-marocain, ce n'est pas uniquement ce que je suis ; je suis également ce que je produis, et je suis, en même temps, ce que je deviens en le produisant ${ }^{12}$.

Dès lors, quelle est la spécificité de la place occupée par la culture afro-marocaine à Essaouira ? Quelle signification donner à ceci, que, à la différence des autres villes marocaines «noires» comme Marrakech -, la population noire d'Essaouira jouit, auprès des autres populations de cette ville, d'une primauté culturelle évidente ? Ce qui fait la spécificité des membres de la communauté noire d'Essaouira, c'est la position particulière qu'ils y occupent comme « guitare du pauvre $»^{13}$ et blues des marges sociales. A Essaouira, les musiciens Noirs sont la guitare du pauvre ou du marginal. Le Gumbri (guitare basse à trois cordes avec caisse rectangulaire d'origine subsaharienne) et le chant représentent le support essentiel de la culture des personnes noires d'Essaouira. En clair, Gumbri et chant sont l'emblème de cette ville. En dépit du statut urbain et social marginal des animateurs de cette musique et de ce répertoire (qui rappelle le pearching des Africains-Américains des ghettos), ce sont des références culturelles acceptées et de plus en plus glorifiées par les jeunes mogadoriens et de plus en plus par des pans entiers de la jeunesse marocaine, voire mondiale. C'est l'effet-diaspora ou l'effet-réseau (Glowczewski 2003).

À Essaouira, la réception «festive» des expressions artistiques et « rituelles » africaines s'est déplacée. Prenons l'exemple du rite : chez les jeunes d'Essaouira, le rite devient une catharsis. Les rites de possession 
africains instituent la ville et, à la fois, celle-ci institue ceux-là. Qu'estce qui explique cette forge métisse qui institue la transversalité existentielle des identités culturelles tant individuelles que collectives? C'est le détournement des arts « nègres » par les «blancs ». Étant indissociables des vicissitudes « post-modernes » du paysage ethnique et social marocain dans son ensemble, les « institutions imaginaires » négromarocaines d'Essaouira sont devenues l'objet d'une captation dont les auteurs sont les clients «blancs » de ces institutions.

En tant que transe des confins de la société, la nuit rituelle de transe (derdeba) des Noirs d'Essaouira incite le marginal ou le « ghettoïsé » à expérimenter les limites de leur transe, à aller au bout de leur transe. Mais, en même temps, cette transe n'est pas mystique; c'est un panaché de fatalité sociale et historique, de sous-développement, de marginalité, de problèmes de jeunesse désœuvrée, de citoyenneté en cours de décomposition-recomposition.

À la marginalité, à la précarité socio-économique et à la fragilité psychologique d'une majorité des jeunes d'Essaouira, il faut ajouter la précarité sociale et infra-structurelle de cette ville : la médina (l'ancienne ville) y est devenue un ghetto, un « bidonville», comme l'atteste l'état de délabrement avancé de l'ancien quartier juif (le mellah).

En bref, les rituels afro-marocains participent d'un contexte social et humain devenu « composite» (le mot est du sociologue marocain Paul Pascon) et non exclusivement de «l'inquiétante étrangeté » de l'imaginaire « africain »d'Essaouira, qui, lui, est d'autant plus fantomatique (dans le sens grec du terme phantasma: fantasmatique et fantomatique) qu'il se nourrit de la mémoire, éteinte, des fastes de jadis, à savoir: l'âge d'or d'Essaouira du temps des négociants juifs, des consuls européens et des soldats de la Garde Noire... Essaouira a cessé d'être le « Port de Tombouctou » et de Marrakech que, jadis, elle fut. Le déclin et la fin, consécutivement à la découverte du Continent américain, du rôle d'intermédiaire commercial transsaharien qui fut, des siècles durant, celui du Maroc entre l'Afrique Noire, le Moyen-Orient et l'Europe, ayant sonné le glas d'un Mogador naguère perçu comme «Eldorado marocain » (Jackson 1820). 


\section{Entre Dionysos, transversalité réflexive, contre-culture et multitude}

Le regard d'une ville croise celui de ses artistes et, au-delà, celui de l'ensemble de ses acteurs. Il faut souligner l'importance de cette synergie existentielle car elle exprime l'impensé (à Essaouira, cet impensé, c'est la précarité) de la ville et, corollairement, ce qu'elle est, ici et maintenant.

L'importance sociale de la culture négro-marocaine à Essaouira est une des conséquences du vide culturel consécutif à la déliquescence de l'ancienne vie culturelle traditionnelle désertée par les jeunes qui, eux, privilégient les groupes folk, qui existent sur les plans national et international et qui sont relayés localement par les musiciens Noirs. De plus, ce phénomène de «blackisation » culturelle renvoie à un statut que, désormais, ces musiciens ont acquis: ils se sont universalisés et, en l'étant devenus effectivement, ils réfléchissent la ville et en sont devenus les interprètes. Ce basculement dans le monde et l'Ailleurs, nous comprenons qu'il symbolise le passage du virtuel au concret: c'est l'effet-réseaux.

En effet, ce rapport réflexif explique ceci, qu'une ville est ce que ses expressions culturelles en disent ; et, en en étant «chantée », décrite, la ville les fait dire et exister, à son tour. D'autre part, et simultanément, les facteurs privilégiés (les musiciens) de ces expressions culturelles sont les aèdes ou les griots de cette situation ethno-urbaine puisqu'ils traduisent -esthétiquement et selon les attendus anthropologiques de l'acte réflexif - cette situation. Inversement, en donnant à voir ou à écouter cette situation, ces expressions culturelles l'approfondissent et l'affirment étant donné que la monstration, ou la manifestation, de ce qu'ily $a$, est indissociable de ce qu'il y $a$, c'est-à-dire, en l'occurrence : la ville, mais également le fait qu'elle est pauvre, délaissée et déshéritée.

La synergie existentielle ville/arts africains renforce la culture noire (musique, chant...) du fait que ces arts sont organiques à la ville et constituent un phénomène irréversiblement urbain. C'est là une situation d'autant plus singulière que l'arrière-pays ou l'espace géographique de référence historiquement attesté des Noirs d'Essaouira et de leurs expressions culturelles et artistiques, c'est l'Afrique au sud du Sahara, et non plus la compagne qui environne cette ville.

Un étrange, mais intense, pacte communicationnel unit les artistes noirs d'Essaouira, - dont l'imaginaire est subsaharien -, à leur public. En étant les révélateurs de l'impensé (la précarité socio-économique) de leur public et de ses conditions matérielles d'existence, ils expriment 
non seulement leurs Ailleurs noirs, mais encore le statut d'oubliés de l'histoire tant de ce public que de la situation altérée, devenue protourbaine, d'Essaouira.

Dans cette situation, les artistes noirs (les Gnawa) constituent l'organon de la quiddité d'une identité culturelle en fusion, en perpétuelle procession vers ce qu'elle est, ici et maintenant. Ces artistes sont les griots de l'étrangeté et de l'absence de fond : ils donnent existence et consistance à la ville et à leur public auxquels ils sont indissociablement liés.

Ce triptyque rhizomatique est la traduction culturelle de la marginalité d'une ville. Extraordinaire communion que ce phénomène de solidarité «esthétique » qui unit les auteurs du Blues négro-marocain, ces troubadours des marges, à un public qui, lui, personnifie une mémoire orale et existentielle des marges de l'histoire. Cette « unitéisme » (Félix Guattari) se fait selon les procédures réglées d'un potlatch inouï : celui où, n'ayant rien à donner, le public offre ses rêves et les musiciens leurs arts et, à travers eux, la catharsis collective et la transe du pauvre.

À Essaouira, les arts noirs constituent, à leur manière, la métaphore vivante du vide culturel et du rêve sans lesquels le public jeune ne peut s'éclater vers ce qu'il est. D'où la transmutation de ce qui n'est jusque-là qu'imaginaire en «fait social total» (Marcel Mauss). Ce fait social total (nous les jeunes, décidons d'exister, ici et maintenant, comme phénomène social surdéterminant) assure le triomphe de l'existence et du rêve sur la fatalité d'une dissociation sociale dont il reste à davantage situer l'origine (Benachir 2005).

Le phénomène artistique afro-marocain est au centre des processus de révélation de l'impensé culturel d'Essaouira. L'influence et l'impact de ce phénomène - et de son universalisation (grâce à la world culture) -, sur les jeunes générations à Essaouira, exprime un phénomène d'acculturation dont la perpétuation est attestée par la reconnaissance dont sont l'objet les emblèmes locaux et internationaux des Ijakane ${ }^{14} \mathrm{de}$ l'étrangeté légitime, de la minorité, des marges de l'humanité, que sont les musiciens noirs ou ceux qui détournent leur art, c'est-à-dire : les «blancs » culturellement blackisés. En clair, les effets « acculturels » qu'inscrivent le cosmopolitisme " phéniste » (qui renaît continûment de ses cendres) et la « délocalisation » planétaire de la culture à possession rituelle et en transes de la communauté noire d'Essaouira, un auteur les résume comme suit : "Étant marginaux, les Gnawa, et plus particulièrement ceux d'Essaouira, ville marginale, ont tendance à attirer dans leur culture toutes les marginalités. » (Lapassade 1998). 
Les musiques noires « chauffeuses » des transes collectives des jeunes générations participent de la manifestation généralisée de l'Autre (l'universalité) et du basculement esthétique du Même (le local) dans l'Autre. Elles contribuent à ce travail du négatif qui fait que la dépossession de soi n'est pas vécue sous le mode de la honte, mais sur celui de l'intentionnalité ou de l'entrée dans le réel concret, dans la vie. Ce phénomène peut s'expliquer par la communauté de situation dont font montre les jeunes à Essaouira consécutivement à l'isolement géographique et à la précarité économique de cette ville. Il peut également l'être par la conservation, même sous une forme quelque peu fragile, de traditions plus fortes finalement dans cette cité que dans une ville comme Marrakech.

\section{Que peut-on préconiser?}

Lévénement de la contribution « citoyenne » à l'élaboration d'un champ politique démocratique est incontournable quant aux noces des peuples et de leur destinée. Et à supposer qu'elle ne soit qu'une ébauche, pareille contribution est un des prolégomènes à une Nouvelle Renaissance du Maroc et de ses socio-cultures. Quoiqu'elle butte sur des contradictions socio-économiques, politiques, théologiques, géostratégiques, etc., internes au Maroc et à ses relations avec le reste du monde (déterminisme de la conjoncture internationale, l'intégrité territoriale), la pensée qui pense cette Nouvelle Renaissance marocaine définit et délimite le redéploiement et l'essor éthique de celle-ci : la démocratie, la liberté, l'unité, la tolérance, la dignité, l'égalité, la communication humaine...

En outre, nous préconisons la prise en compte de quelques perspectives majeures. Pour que se réalise un Maroc politiquement et culturellement multiple, il est nécessaire et urgent pour ce pays de prendre en compte quelques perspectives majeures parmi lesquelles le courage salubre de devenir : - un horizon de rationalité, - un horizon de dignité, - un horizon de liberté, - un horizon diversaliste. En effet, ces horizons fondent l'égalitarisme et, partant, la démocratie. Ceci d'une part. D'autre part, ils ne sont pas créateurs de Limes à l'intérieur et autour du Maroc dont le paradigme de région est porteur de la portée fédérale ou confédérale de l'État marocain d'autant plus que la dimension culturelle de chaque région montre comment chacune d'elles, cons- 
titue, effectivement et depuis des millénaires, une région à histoire et à écriture.

\section{Notes}

1 À ce propos, voir la Déclaration de l'UNESCO sur la diversité humaine et culturelle : www.unesco.org/déclaration.

2 Cinq périodes marquent la transition politique au Maroc : VIIIe-XIVe siècle, XIVe début du XXe siècle, 1912-1956 (période des protectorats français et espagnol), et 1956-1992-94.

3 Il va de soi que nous ne nous intéressons pas, ici, aux conséquences catastrophiques que ne manqua pas d'avoir, des siècles durant, le rapport militariste de l'État à la société concrète. D'autre part, l'étude ici proposée ne prend en considération, ni les causes du non avènement du « citoyen », ni les « appareils idéologiques » qui ont servi l'État que voilà, tels la casuistique fikhique (théojurisconsulte), les confréries religieuses (zaouia), le mysticisme «savant» et ses «transcendances », l'inconscience des acteurs sociaux dans l'usage qu'ils font du langage (terminologie, sémantique, concepts) comme outil cognitif rationnel dans son rapport à la réalité, l'absence tragique d'une culture esthétique et éducative qui initie l'enfant, mais également la femme-mère, aux ars vitce (dans le sens que Michel Foucault a décisivement imprimé à ce terme). En clair, notre propos n'est pas de « déconstruire » les effets ravageurs induits par la destruction post-averroèssiennes de la raison par le «théologien » Hamid al-Ghazali.

4 Sur la « réforme » au Maroc, cf. Hespéris Tamuda, Vol. XXXIX, Fasc. 2, Rabat : Université Mohammed V, Faculté des Lettres et des Sciences humaines, 2001 : 7-108.

5 Voir, infra, la section La région entre invention, création et résistances.

6 Voir Rosanvallon, Pierre, 2002 « Pour une histoire du politique », in Le Monde, Paris, 30 mars : 16. L'auteur y dit, en substance, ceci, que le fait que la démocratie soit une institution faite pour l'expérimentation de la liberté, elle ne suffit pas pour autant pour qu'elle soit une solution problématique au service de l'instauration de la «Cité » qu est, elle-même, au service d'hommes libres.

7 Quand elle intègre à sa logosphère les sociétés maghrébines, la littérature panarabe relative à la Renaissance est plus soucieuse de l'«unité » de la «nation » arabe et de son accès à la « modernité » que de la démocratie comme horizon indépassable de l'existence humaine. $C f$., à titre d'exemple, Moussa 1987, Ghalioun 1987, Al-Jabri 1988, 1995.

8 Bekri, Tahar, 1994 : 206 : «Et c'est en empêchant la plume lumineuse que le sabre a transformé la majorité de nos pays en ruines où les traces du sang aveuglent nos utopies généreuses et nos idéaux animés de justice et de fraternité. Qui emprisonné, qui banni, qui contraint à l'exil, qui exilé dans son propre pays, la liste serait longue de tous les écrivains blessés ou morts sous le sabre, la volonté de l'État, monopole du dictateur aidant. » 
9 Le retour en force du religieux dans les ambitions, les visions et les utopies de tout un peuple ne sont pas propres aux seuls peuples des pays susmentionnés. L'exemple nord-américain est une illustration « post-moderne » très significative.

10 À titre illustratif, voir, Daoui, Ali, 2003, «L'identité culturelle maghrébine et la question de la légitimité », La question de la culture dans le Maghreb arabe, in At-Takafa al-Maghribia, $\mathrm{n}^{\circ}$ 22/23, Rabat, Royaume du Maroc, Ministère de la culture : 78-96, notamment note 31, p. 96 (en arabe).

11 Du 12 décembre 1962 au 5 novembre 1997, tous les discours de feu le roi Hassan II accordent une place privilégiée au concept de Région. Ainsi en est-il des discours du 12-12-1962, du 8-10-1963, du 3-3-1967, du 9-10-1967, du 12-9-1968, du 22-3-1969, du 24-10-1984, du 8-4-1988, du 24-7-1989, du 89-1992, du 16-9-1992, du 3-3-1993, du 29-3-1993, du 25-5-1993, du 16-71993, du 9-4-1994, du 6-2-1994, du 3-3-1996, du 20-8-1996, du 5-1 1-1996, et du 5-11-1997. De même le discours de S.M. Le Roi Mohammed VI, prononcé le 6 novembre 2003 ainsi que les discours récents présentant l'INDH (Initiative Nationale de Développement Humain, été 2005), accordent une place d'importance multidimensionnelle à la Région.

12 C'est tout le contraire de l'identité individuelle de l'artiste marocain d'origine bédouine, ou rurale, déplacé, sub-urbanisé, du fait de l'exode rural. Nous l'avons déjà noté, Essaouira est, dès sa fondation, une ville, au sens plein du terme. De là, chez les Noirs de cette cité, la constitution, entre autres éclatements vers le réel, d'un rapport esthético-urbain à la réalité et l'existence.

13 Pour détourner l'expression du sociologue américain Irving Goffman: «Culture du pauvre».

14 Terme amazigh-berbère signifiant nomades, passeurs.

\section{Bibliographie}

Al-Jabri, Mohammed Abed, 1988, Le discours arabe contemporain, Essai d'analyse critique, Beyrouth : Éd. Dar Attaliaâ, $3^{\mathrm{e}}$ édition.

Al-Jabri, Mohammed Abed, 1995, Introduction à la critique de la raison arabe, Casablanca : Le Fennec.

Allam, Azzeddine, 2005, « De la sujétion à la citoyenneté, Itinéraire du concept de Ra'iya». (Texte intégral de la contribution de l'auteur aux travaux du Colloque du CODESRIA, Maputo, décembre 2005).

Amselle, Jean-Loup, 1990, Logiques métisses: anthropologie de l'identité en Afrique et ailleurs, Paris : Éd. Payot, Édition revue et augmentée en 1999, chez le même éditeur.

Amselle, Jean-Loup, et M’Bokolo, Elikia, 1985, rééd. 1999, Au cæur de l'ethnie: ethnies, tribalisme et État en Afrique, Paris : Éd. La Découverte.

An-Naçiri Es-Slaoui, Ahmed Ben Khaled, à partir de 1923, Kitab El-Istiqça li-Akhbar El-Maghrib el-Aqça, tr. fr. Histoire du Maroc, plusieurs vol., Paris : Geuthner. Notamment le vol. IX.

Bariton, L., 1995, Les grandes lignes de l'histoire des Chichaoua, Paris : CREAM, n 1234. 
Bekri, Tahar, 1994, Littératures de Tunisie et du Maghreb, Paris : L'Harmattan.

Benachir, Bouazza, 2001, Négritudes du Maroc et du Maghreb. Servitude, cultures à possession et transthérapie, préface de G. Lapassade, Paris : L'Harmattan,.

Benachir, Bouazza, 2005, Esclavage, diaspora africaine et communauté Noire du Maroc, préface de René Schérer, Paris : L'Harmattan.

Ben Abdallah El Alaoui, Hicham, 2005, « Crise et réforme dans le monde arabe », in Le Journal hebdomadaire, Casablanca, 7 octobre.

Ben Driss Ottmani, Dris, 1997, Une cité sous les alizés. Mogador, des origines à 1939, Rabat: La Porte.

Benoit, F., 1933, Cornut, l'Avignonnais et l'urbanisme à Mogador au XVIIIe siècle, Mémoire de l'Institut Historique de Provence, $3^{\mathrm{e}}$ trim

Berque, Jacques, 1974, Langages arabes du présent, Paris : Gallimard.

Crapanzano, Vincent, 1973, The Hamadscha, Berkley : Univ. of California Press.

Chlyeh, Abdehafid, 1999, Les Gnaoua du Maroc, Grenoble : La Pensée sauvage.,

Chottin, Alexis, 1938, Tableau de la musique marocaine, Paris : Paul Geuthner.

Coulon, Alain, 1987, L'Ethnométhodologie, Paris : PUF, Que sais-je?.

Daoui, Ali, 2003, «L'identité culturelle maghrébine et la question de la légitimité », in La question de la culture dans le Maghreb arabe (dossier spécial), in At-Takafa alMaghribia, $\mathrm{n}^{\circ}$ 22/23, Rabat, Ministère de la Culture (en arabe).

Daoui, Ali, 2003, «Lidentité culturelle maghrébine et la question de la légitimité », in La question de la culture dans le Maghreb arabe, in At-Takafa al-Maghribia, ${ }^{\circ} 22 /$ 23, Rabat, Royaume du Maroc, Ministère de la culture : 78-96, notamment note 31 : 96 (en arabe).

Deleuze, Gilles et Guattari, Félix, 1980, Mille Plateaux, Capitalisme et schizophrénie II, Paris : Minuit.

Dermenghem, Émile, 1954 (rééd. 1982), Le Culte des saints dans l’islam maghrébin, Paris : Gallimard.

Etchegoyen, Alain, 1993, La Démocratie malade du mensonge, Paris : François BourtinJulliard.

Ennaji, Mohammed, 1994, Soldats, domestiques et concubines. L'esclavage au Maroc au XIXème siècle, Casablanca-Paris : Balland/Le Nadir.

Garfinkel, Harold, 1967, 1984), Studies in Ethnomethodology, Englewood Cliffs N.J. : Prentice Hall (2e édition 1984, Cambridge : Polity Press).

Ghalioun, Bourhan, 1987, Qu'est-ce que la renaissance ?, Alger : Maoufam Li Annachr (en arabe).

Glowczewski, Barbara, 2003, «La pensée en réseau des Aborigènes », in Lévi-Strauss et la pensée sauvage, Le Nouvel Observateur, Hors-Série n 51 , Paris, juillet/août.

Herzenni, Ahmed, 2002 Écrits sur la gauche, l'islam et la démocratie (en arabe), s.l. : Éditions de la Société Oudad de Communication.

Hespéris Tamuda, 2001,Vol. XXXIX, Fasc. 2, Rabat, Université Mohammed V, Faculté des Lettres et des Sciences humaines(dossier spécial : La réforme et ses usages. Perspectives marocaines).

Höst, Georg, 1779, 1998, Histoire de l'Empereur du Maroc Mohammed ben Abdallah, tr. fr. F. Damgaard et P. Gailhanou, Rabat : La Porte (édition originale en danois, 1779). 
http://www.unesco.org/déclaration.

Jackson, J.Greg, 1820, An Account of the Empire of Marocco \& the district of Suse and Tafilelt, London, tr. fr. Robinet, Jean-François, 2005, Relation de l'Empire du Maroc. Description du Maroc, de la Côte ouset-africaine et de Tombouctou (1789-1805, présentation de Mohamed El Mansour, Rabat : Institut des Études Africaines, Université Mohammmed V-Souisssi.

Jodin, André, 1967, Mogador, comptoir phénicien au Maroc atlantique, Tanger : Éd. Marocaines et internationales.

Julien, Charles-André, 1951, Histoire de l'Afrique du Nord, Paris : Payot.

Kahn, Pierre, 1989, L'État, Paris : Éditions Quintette.

Leveau, Rémi et Hammoudi, Abdellah (sous la dir.), et Mohsen-Finan, Khadija (coordination), 2004, Monarchies arabes (Transitions et dérives dynastiques), Paris : IFRI, col. « Les études de la Documentation française ».

Lapassade, Georges, 1969, «Essaouira, ville à vendre », in Lamalif, nº 33, Casablanca.

Lapassade, Georges, 1982, Gens de l'ombre, Transes et Possessions, Anthropos, Paris.

Lapassade, Georges, 1993, L'ethnosociologie, Paris: Méridiens-Klincksieck.

Lapassade, Georges, 1994, « Notes sur l'histoire de Mogador », in Traces du Présent, $\mathrm{n}^{\circ}$ 2-3, Marrakech.

Lapassade, Georges, 1998, «Les Gnaoua d'Essaouira, thérapeutes de la différence », in Africultures, Paris : L'Harmattan, octobre.

Laroui, Abdellah, 1977, Les origines sociales et culturelles du nationalisme marocain (18301912), Paris, Maspero. Rééd. Casablanca, Centre culturel arabe, 1993 (réédition).

Laroui, Abdellah, 1981, Mafhum ad-dawla (Le concept d'État), ch. V, Centre culturel arabe, Casablanca (en arabe).

Laroui, Abdellah, 1987, Islam et modernité, Paris : La Découverte.

Mana, Abdelkader et Delaborde, Philippe, 1991, Le temps d'une ville : Essaouira, Casablanca : EDDIF (s.p.).

Miège, Jean-Louis, 1999, « De quelques remarques historiques à propos des Gnaoua d'Essaouira », in L'univers des Gnaoua (sous la dir. de A. Chlyeh), CasablancaGrenoble : Le Fennec-La Pensée sauvage.

Monji, Maâti, 2004, La monarchie marocaine, Paris : L'Harmattan.

Montagne, Robert, 1989, Les Berbères et le Makhzen dans le sud du Maroc, Rabt, Archives marocaines. Rééd. Casablanca, Afrique/Orient.

Moussa, Salama, 1987, Qu'est-ce que la renaissance?, Alger : Maoufam Li Annachr (en arabe).

Nassar, Nassif, 1997, «Problèmes des limites entre raison et idéologie », in La raison et la question des limites (Ali Benmakhlouf dir.), Casablanca : Éditions Le Fennec : 57-80 (en arabe).

Pâques, Viviana, 1964, 1995, L'Arbre cosmique dans la pensée populaire et dans la vie quotidienne du Nord-Ouest africain, Paris : CNRS. Rééd. 1995, Paris : L'Harmat$\tan$.

Pâques, Viviana, 1990, La religion des esclaves. Recherches sur la confrérie marocaine des Gnawa, Bergamo : Moretti \& Vitali editori. 
Quéré, Louis, 1984, «L'argument sociologique de Garfinkel », in Arguments ethnométhodologiques, Problèmes d'épistémologie en sciences sociales, III, Centre d'études des mouvements sociaux, Paris : EPHSS-CNRS.

Rachik, Hassan, 2001, "Jma'a, tradition et politique », in Hespéris Tamuda, Vol. XXXIX, Fasc. 2, Rabat, Université Mohammed V-Agdal, Faculté des Lettres et des Sciences humaines : 14-156.

Robinet, Jean-François, 1996, Esquisses pour Essaouira, Traces du Présent, Marrakech.

Rovsing Olson, Myriam, 1997, Chants et danses de l'Atlas (Maroc), Cité de la Musique/Actes Sud.

Rosanvallon, Pierre, 2002 « Pour une histoire du politique », in Le Monde, Paris, 30 mars.

Roussillon, Alain, 2001, «La réforme et ses usages, Perspectives marocaines », in Hespéris Tamuda, Vol. XXXIX, Fasc. 2, Rabat: Université Mohammed V, Faculté des Lettres et des Sciences humaines.

Schutz, Alfred, 1987 (1932 pour la première édition), Le chercheur et le quotidien, Paris : Méridiens Klincksieck.

Sedrati, Ali, 1995, «La région au Maroc entre la légitimité politique et la complexité économique », in Région et développement économique, t. III, Rabat : Premier Ministre, Ministère chargé de la population et Faculté d'Économie Appliquée (Centre d'Économie Régionale), Université d'Aix-Marseille, 19 et 20 octobre : $111-117$.

Terrasse, Henri, 1950, Histoire du Maroc, t. 2, Casablanca : Éditions Atlantides.

Tremearne, A. J. N., 1991, The Ban of the Bori: demons and demon-dancing in West and North Africa, London (reprint, Paris, Maisonneuve-Geuthner).

Tremearne, A. J. N., 1913, Hausa Superstitions and Customs, London.

Troin, Jean-François, 1975, Les souks marocains. Marchés ruraux et organisation de l'espace dans la moitié nord du Maroc, Aix-en-Provence : Edisud, 2 volumes.

Tuéni, Ghassan, 1991, Kiraatoun Thaniatoun fi al-Qaoumia al-Arabia (en arabe), Deuxième lecture du nationalitarisme arabe, Beyrouth.

Vairel, Frédéric, 2001, «L'année 1993 : Infortunes d'un dessein royal et paroles publiques », in Hesperis Tamuda, Vol. XXXIX, Fasc. 2, Rabat : Université Mohammed V, Faculté des Lettres et des Sciences humaines.

Westermarck, E., 1926 (1 re édition), 1968, Ritual and Belief in Morocco, New York: University Books, Introduction by B. Malinowski, London, Macmillan.

Westermarck, E., 1934, « Negro Influence in Morocco », in Negro Anthology (Nancy Cunard dir.), London.

Wijhat Nadhar (revue), 2001, «La laïcité et la question de la transition démocratique au Maroc, Espaces consensuels »(dossier spécial, en arabe), in $\mathrm{n}^{\circ} 10$, Rabat, automne.

Wijhat Nadhar (revue), 2004 «L'agonie de la transition démocratique au Maroc » (dossier spécial, en arabe), in $n^{\circ} 23$, Rabat, automne. 